\title{
Downregulation of RNF128 Predicts Progression and Poor Prognosis in Patients with Urothelial Carcinoma of the Upper Tract and Urinary Bladder
}

\author{
Yi-Ying Lee ${ }^{1,2}$, Chieh-Tien Wang1,3, Steven Kuan-Hua Huang, Wen-Jeng Wu ${ }^{5,6,7}$, Chun-Nung Huang5,6, \\ Ching-Chia Li5,6,7, Ti-Chun Chan², Peir-In Liang8, Chung-Hsi Hsing9 ${ }^{9}$, Chien-Feng Li10,11,12,13凶 \\ 1. Department of Pathology, Chi Mei Medical Center, Liouying, Taiwan; \\ 2. Institute of Biomedical Science National Sun Yat-sen University, Kaohsiung, Taiwan; \\ 3. Department of Medical Laboratory Science and Biotechnology, Chung Hwa University of Medical Technology; \\ . Department of Urology, Chi Mei Medical Center; Tainan, Taiwan; \\ 5. Department of Urology, Faculty of Medicine, Kaohsiung Medical University; \\ 6. Department of Urology, Kaohsiung Medical University Hospital, Kaohsiung Medical University; \\ Department of Urology, Kaohsiung Municipal Ta-Tung Hospital; \\ Department of Pathology, Kaohsiung Medical University Hospital, Kaohsiung Medical University; \\ Department of Anesthesiology, Chi Mei Medical Center, Tainan, Taiwan \\ 10. Division of Clinical Pathology, Chi Mei Medical Center, Tainan, Taiwan; \\ 11. National Institute of Cancer Research, National Health Research Institutes, Tainan, Taiwan; \\ 12. Department of Biotechnology, Southern Taiwan University of Science and Technology, Tainan, Taiwan; \\ 13. Institute of Clinical Medicine, Kaohsiung Medical University, Kaohsiung, Taiwan. \\ $\triangle$ Corresponding authors: Chien-Feng Li, M.D., Ph.D. Department of Pathology, Chi Mei Medical Center. Chunghwa Road, Yung Kang Dist., Tainan, 710, Taiwan. Phone: \\ +886-6-2812811 ext. 53680, Fax: +886-6-2511235 E-mail: angelo.p@yahoo.com.tw; or Wen-Jeng Wu, M.D., Ph.D. Department of Urology, Kaohsiung Medical University \\ Hospital. No. 100, Tzyou 1st Road, Kaohsiung, 807, Taiwan. Tel. +886-7-3208212; Fax: +886-7-3211033. Email:wejewu@kmu.edu.tw.
}

(C) Ivyspring International Publisher. Reproduction is permitted for personal, noncommercial use, provided that the article is in whole, unmodified, and properly cited. See http://ivyspring.com/terms for terms and conditions.

Received: 2016.07.11; Accepted: 2016.09.04; Published: 2016.10.25

\begin{abstract}
Background: The TP53 tumor suppressor gene plays a crucial role in the carcinogenesis of many malignancies, including urothelial carcinoma (UC). Overexpression of $p 53$ is associated with poor prognosis in UC. Recently, RING finger protein 128 (RNF128) was shown to be involved in p53-induced apoptosis, forming a negative feedback loop. However, the significance of RNF128 in patients with UC remains unknown. In this study, our aim was to evaluate the expression of RNF128 in UC and to assess its predictive and prognostic value in a well-established cohort.

Methods: Through data mining from a published transcriptome (GSE31684), RNFI28 was identified as the most differentially expressed gene in UC among those associated with negative regulation of the cytokine biosynthetic process (GO:0042036). Its immunoexpression was further evaluated using the H-scores of 340 patients with upper urinary tract UC (UTUC) and 295 with urinary bladder UC (UBUC). The scores were correlated with clinicopathological features, disease-specific survival (DSS) and metastasis-free survival (MeFS). We also used Western blot analysis to evaluate RNF128 protein expression in human urothelial cell (HUC) lines.

Results: Downregulation of RNF128 expression was significantly associated with advanced $\mathrm{pT}$ stage $(p<0.001$ ), high histological grade (UTUC, $p<0.001$; UBUC, $p=0.035$ ), nodal metastasis (UTUC, $p<0.001$; UBUC, $p=0.001$ ), vascular invasion (UTUC, $p<0.001$; UBUC, $p=0.008$ ) and high mitotic rate (UTUC, $p=0.003$; UBUC, $p=0.023$ ). Low expression of RNF1 28 was an adverse prognosticator for DSS (UTUC, $p<0.0001$; UBUC, $p<0.0001$ ) and MeFS (UTUC, $p<0.0001$; UBUC, $p=0.0002$ ). Moreover, low expression was predictive of poor DSS (UTUC, $p=0.006$; UBUC, $p=0.003$ ) and MeFS (UTUC, $p=0.009$; UBUC, $p=0.036$ ) in multivariate comparisons. Western blot analysis showed that the RNF128 protein was downregulated in invasive urothelial cancer cell lines.

Conclusion: Our findings showed that downregulation of RNF128 was correlated with cancer invasiveness and metastasis as well as reduced survival in patients with UTUC and UBUC, identifying RNF128 as a prognostic factor in UC.
\end{abstract}

Key words: RNF128, Grail, urothelial carcinoma, prognosis. 


\section{Introduction}

Urinary bladder cancer is the ninth most common malignancy worldwide, and the common histological type is urothelial carcinoma (UC) [1-3]. Although cisplatin-based systemic chemotherapy is used to treat advanced diseases, urinary bladder urothelial carcinoma (UBUC) still ranks 13th as a cause of cancer death [2]. More than $90 \%$ of UCs arise in the urinary bladder, and only $5 \%$ to $10 \%$ of all UCs develop in the upper urinary tract [1-3]. Although growing evidence has suggested that UBUC and upper urinary tract UC (UTUC) may share several biologically unique features, there are certain significant similarities between both types of UCs [4-9]. Additionally, it was reported that there was a $30-50 \%$ risk of bladder recurrence within 5 years for patients with UTUC receiving radical nephroureterectomy and a 2-6\% risk of developing subsequent UTUC after initial diagnosis with UBUC [10-11]. Furthermore, the behavior of both diseases is identical after adjusting for tumor stage and histological grade [6-7]. We therefore hypothesized that both UTUC and UTUB may share similar tumorigenesis pathways.

Regardless of the inciting mechanism and/or long-term behavior, further elucidation of the molecular basis of UBUC and UTUC will have important implications in understanding the differences or similarities in the genetic profiles as well as identifying prognostic biomarkers to develop novel targeted therapy. To address this, we performed data mining from a published transcriptome of UBUC (GSE31684), identifying RING finger protein 128 (RNF128) as the most significant gene for cancer invasiveness and metastasis among those associated with negative regulation of the cytokine biosynthetic process (GO:0042036).

RNF128 (also known as Grail, a gene related to anergy in lymphocytes) is an E3 ubiquitin ligase functioning as a regulator of anergy and cytokine production [12-14]. It was shown to play an important role in controlling Th2 development through a negative feedback loop [13-14]. A recent report found that RNF128 was a p53-interacting glycoprotein and may be crucial in p53-induced apoptosis under variable stress conditions [12]. The transcription factor p53, a well-known tumor suppressor encoded by the TP53 gene, regulates genes critical for cell cycle arrest, senescence and apoptosis to maintain genome stability [15-17]. TP53, the most frequently mutated gene, has been identified in approximately $50 \%$ of all human cancers [15-19]. Moreover, p53 is one of the most commonly studied markers in UC [20-22].

While the crucial role that p53 plays in cancers is apparent, the involvement of RNF128 in tumorigenesis remains unknown, and little if any research has investigated its function [12]. To our knowledge, this is the first study to elucidate the relationship between RNF128 expression and clinical presentation in patients with UBUC and UTUC. We also aimed to comprehensively analyze its expression and associated clinicopathological factors as well as disease survival in our well-characterized cohort of UC patients.

\section{Materials and Methods}

\section{Analysis of a public transcriptomic dataset of UC}

To identify relevant genes that are critical in the pathogenesis of UC, we performed data mining on GEO (National Center Biotechnology Information) and identified a dataset (GSE31684) which profiled radical cystectomy specimens from 93 patients with UBUC using an Affymetrix U133 Plus 2.0 Array. To analyze the gene expression levels, we imported the raw CEL files into Nexus Expression 3 (BioDiscovery, EI Segundo, CA, USA). All probe sets were tested without preselection or filtering. Supervised comparative analysis and functional profiling were carried out to identify statistically significant genes that were associated with negative regulation of the cytokine biosynthetic process (GO:0042036). The statistical significance of differentially expressed transcripts was determined, and transcripts were chosen to validate on the basis of primary tumor (pT) status and the development of metastatic events. We performed a detailed comparison between the differential expression in low-stage (pTa-pT1) UCs and high-stage (pT2-pT4) UCs as well as UC with and without distal metastasis. All cases were dichotomized into two groups, high- and low-expression, to assess the prognostic impact of selected gene using survival analyses.

\section{Patient characteristics and tumor specimens}

A total of 635 UC patients (340 UTUCs and 295 UBUCs) were enrolled as the subjects of the study, and the data were retrieved from the archives of Chi-Mei Medical Center between 1996 and 2004. Other histological variants and synchronous UTUC and UBUC were excluded. The study protocol and the paraffin-embedded tissue blocks used for immunohistochemistry were approved by the institutional review board (IRB10302015). All patients with UTUC underwent nephroureterectomy with bladder cuff excision and regional lymph node (LN) dissection, while those with superficial UBUC (pTa-pT1) received transurethral resection of the bladder tumor (TUR-BT), with or without subsequent 
intravesical BCG injection. Radical cystectomy was performed as a salvage treatment for UBUC recurrence. For muscle-invasive UBUC, radical cystectomy with bilateral pelvic LN dissection was performed. Patients who had advanced UBUC disease, i.e., pT3 or pT4 and/or LN metastasis, were treated with systemic adjuvant chemotherapy after the surgical removal of all gross lesions. In comparison, only 29 of the 106 UTUC patients with pT3 or pT4 stage and/or nodal involvement received adjuvant chemotherapy. The criteria for the clinicopathological evaluation were essentially identical to our previous studies [23-28].

\section{Real-time RT-PCR}

For quantification of RNF128 transcript levels in urothelial cells, total RNAs from urothelial cancer cells were extracted for reverse-transcription PCR. Pre-designed TaqMan probes targeting RNF128 (Hs00226053_m1) were used to measure the mRNA levels with an ABI StepOnePlus ${ }^{\mathrm{TM}}$ System as described in our previous studies [26, 28]. After normalization to the internal control of POLR2A (Hs01108291_m1), the expression of RNF128 relative to that of normal urothelium was determined by the comparative Ct method.

\section{Immunohistochemical staining and scoring of RNF 128}

For immunohistochemistry, paraffin-embedded tissue blocks were sectioned at a thickness of $4 \mu \mathrm{m}$,

deparaffinized, and rehydrated. Subsequently, the sections were incubated with a primary antibody targeting RNF128 (1:200, Rabbit monoclonal [EPR7426(2)], Abcam) for an hour after quenching endogenous peroxidase activity with saline for 15 minutes. RNF128 immunostaining was examined by two independent pathologists (P.I.L and C.F.L), who were unaware of the patients' clinicopathological characteristics. Scoring of RNF128 immunoreactivity was determined based on a combination of the percentage and intensity of positive cytoplasmic staining to obtain the H-score, which was calculated using the following equation: $\mathrm{H}$-score $=\Sigma \mathrm{Pi}(\mathrm{i}+1)$. In this formula, $i$ represents the intensity of the stained tumor cells $(0-3+)$, and $\mathrm{Pi}$ is the percentage of stained tumor cells for each intensity varying from $0 \%$ to $100 \%$, with an obtained score ranging from 100-400. The score 100 indicated that $100 \%$ of tumor cells were negative, and 400 indicated that $100 \%$ of tumor cells showed an intense, strong staining (3+) [23-28]. Concordance for interpretation of RNF128 between the two pathologists was above $90 \%$. For those with discrepancies of the histological slides, cases were then independently reanalyzed, resulting in consensus scores.

\section{Cell lines and culture conditions}

Four UC cell lines, RT4, RT9 (formally known as RTCC-1/KMC), J82, and TSGH8301, were grown based on the suggested media and conditions as described in our previous research studies $[29,30]$.

\section{Western blot assays}

Equal amounts of total cellular protein $(25 \mu \mathrm{g})$ extracts were separated on $4 \%-12 \%$ gradient sodium dodecyl sulfate polyacrylamide gels, transferred to polyvinylidene difluoride membranes and then probed with antibodies against RNF128 (1:1000, Rabbit monoclonal [EPR7426(2)], Abcam) with protocols identical to our previous publications [28].

\section{Statistical analysis}

The statistical software package (V.14.0, SPSS Inc., Chicago, Illinois, USA) was used for all data entry and analysis. The median H-score of RNF128 was used as the cut-off to dichotomize the study cohort, separating cases into high- and low-expression groups. The chi-square test was used to compare the RNF128 expression status and the clinicopathological variables tested. The end points analyzed were disease-specific survival (DSS) and metastasis-free survival (MeFS), calculated from the starting date of resection of the tumors to the date of event developed. Patients lost to follow-up were censored on the latest follow-up date. Kaplan-Meier plots and the log-rank test were used to detect significant differences in univariate survival analyses. Those parameters with univariate $\mathrm{p}<0.1$ were included in multivariate analyses using Cox proportional hazards regression. For all analyses, two-sided tests were performed, with $\mathrm{p}<0.05$ set as significant.

\section{Results}

RNF128 identified as a significant differentially downregulated transcript involved in dysregulation of cell growth in UBUC

From the transcriptomic profile, 7 probes covering 3 transcripts associated with negative regulation of the cytokine biosynthetic process (GO:0042036) were analyzed. RNF128 was the only significant gene that met the study selection criteria (Figure 1 and Table 1). Downregulation of RNF128 showed a significant association with primary tumor status increases $\left(\log _{2}\right.$ ratio, $\left.-2.385 ; \mathrm{p}<0.0001\right)$ and distal metastasis $\left(\log _{2}\right.$ ratio, $\left.-1.1327 ; \mathrm{p}=0.0093\right)$. In light of these findings, we were prompted to investigate endogenous RNF128 levels and their clinical significance in our well-characterized UC cohort. 


\section{Clinicopathological findings in UTUC and UBUC}

Table 2 presents the clinicopathological findings of the UTUC and UBUC cases. While the UTUC patients showed relatively even distribution across both sexes, male sex accounted for the majority of the UBUC patients $(n=216,73.2 \%)$. Most cases in the UTUC and UBUC groups were older than 65 years $(n=202,59.4 \%$ for UTUC; $n=174,60.0 \%$ for UBUC). In the UTUC group, a total of 62 patients $(18.2 \%)$ had multifocal diseases, 49 (14.4\%) of which presented with synchronous tumor involvement of the renal pelvis and ureter. Advanced pT stages (pT2-pT4) were identified in $159(46.8 \%)$ and $123(41.7 \%)$ of the UTUC and UBUC cases, respectively. Nodal involvement was identified in $28(8.2 \%)$ and 29 $(23.6 \%)$ of the UTUC and UBUC patients, respectively. More than $80 \%$ of the UC patients in both groups had a high histological grade $(n=284$, $83.5 \%$ for UTUC; $n=239,81.0 \%$ for UBUC). Other relevant histological findings of significance including vascular and perineural invasion as well as a high mitotic rate. A more detailed description of the results is provided in Table 2 .

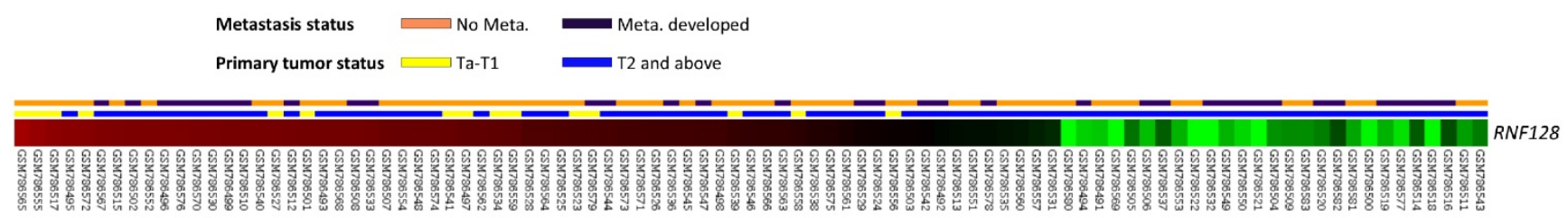

Figure 1. Data mining on a public transcriptomic dataset of urothelial carcinoma. By reappraising the published transcriptomic dataset of urinary bladder urothelial carcinoma (GSE31684), RNF128 is identified as the most significantly downregulated gene associated with cancer invasiveness and metastasis in clustering analysis focusing on cytokine biosynthetic process (GO:0042036). Specimens from high pT (blue lines) and low PT (yellow lines) are indicated on top of the heatmap, and upregulation and downregulation of genes are illustrated as a spectrum of brightness of red and green, respectively. Those with unaltered mRNA expression are coded as black.

Table 1. Summary of differentially expressed genes associated with negative regulation of cytokine biosynthetic process (GO:0042036) and showed positive associations to cancer invasiveness and metastasis in the transcriptome of urinary bladder urothelial carcinoma (GSE31684).

\begin{tabular}{|c|c|c|c|c|c|c|c|}
\hline \multirow[t]{2}{*}{ Probe } & \multicolumn{2}{|c|}{ Comparing T2-4 to Ta-T1 } & \multicolumn{2}{|c|}{ Comparing Meta. to Non-Meta." } & \multirow{2}{*}{$\begin{array}{l}\text { Gene } \\
\text { Symbol }\end{array}$} & \multirow[t]{2}{*}{ Gene Title } & \multirow[t]{2}{*}{ Molecular Function } \\
\hline & $\log$ ratio & p-value & $\log$ ratio & p-value & & & \\
\hline 219263_at & -2.385 & $<0.0001$ & -1.1327 & 0.0093 & RNF128 & $\begin{array}{l}\text { RING finger } \\
\text { protein } 128\end{array}$ & $\begin{array}{l}\text { ligase activity, metal ion binding, protein binding, } \\
\text { ubiquitin-protein ligase activity, zinc ion binding }\end{array}$ \\
\hline
\end{tabular}

Table 2. Correlations between RNF128 Expression and other important clinicopathological parameters in urothelial carcinomas.

\begin{tabular}{|c|c|c|c|c|c|c|c|c|c|}
\hline \multirow[t]{3}{*}{ Parameter } & \multirow[t]{3}{*}{ Category } & \multicolumn{4}{|c|}{ Upper Urinary Tract Urothelial Carcinoma } & \multicolumn{4}{|c|}{ Urinary Bladder Urothelial Carcinoma } \\
\hline & & \multirow[t]{2}{*}{ Case No. } & \multicolumn{2}{|c|}{ RNF128 Expression } & \multirow[t]{2}{*}{ p-value } & \multirow[t]{2}{*}{ Case No. } & \multicolumn{2}{|c|}{ RNF128 Expression } & \multirow[t]{2}{*}{ p-value } \\
\hline & & & High & Low & & & High & Low & \\
\hline \multirow[t]{2}{*}{ Gender } & Male & 158 & 78 & 80 & 0.277 & 216 & 104 & 112 & 0.339 \\
\hline & Female & 182 & 92 & 90 & & 79 & 43 & 36 & \\
\hline \multirow[t]{2}{*}{ Age (years) } & $<65$ & 138 & 68 & 70 & 0.825 & 121 & 64 & 57 & 0.380 \\
\hline & $\geq 65$ & 202 & 102 & 100 & & 174 & 83 & 91 & \\
\hline \multirow[t]{3}{*}{ Tumor location } & Renal pelvis & 141 & 67 & 74 & 0.727 & - & - & - & - \\
\hline & Ureter & 150 & 77 & 73 & & - & - & - & - \\
\hline & Renal pelvis \& ureter & 49 & 26 & 23 & & - & - & - & - \\
\hline \multirow[t]{2}{*}{ Multifocality } & Single & 278 & 140 & 138 & 0.779 & - & - & - & - \\
\hline & Multifocal & 62 & 30 & 32 & & - & - & - & - \\
\hline \multirow[t]{3}{*}{ Primary tumor $(\mathrm{T})$} & $\mathrm{Ta}$ & 89 & 69 & 20 & $<0.001^{*}$ & 84 & 57 & 27 & $<0.001^{*}$ \\
\hline & $\mathrm{T} 1$ & 92 & 55 & 37 & & 88 & 49 & 39 & \\
\hline & $\mathrm{T} 2-\mathrm{T} 4$ & 159 & 46 & 113 & & 123 & 41 & 82 & \\
\hline \multirow[t]{2}{*}{ Nodal metastasis } & Negative (N0) & 312 & 167 & 145 & $<0.001^{*}$ & 266 & 141 & 125 & $0.001^{*}$ \\
\hline & Positive (N1-N3) & 28 & 3 & 25 & & 29 & 6 & 23 & \\
\hline \multirow[t]{2}{*}{ Histological grade } & Low grade & 56 & 45 & 11 & $<0.001^{*}$ & 56 & 35 & 21 & $0.035^{*}$ \\
\hline & High grade & 284 & 125 & 159 & & 239 & 112 & 127 & \\
\hline \multirow[t]{2}{*}{ Vascular invasion } & Absent & 234 & 138 & 96 & $<0.001^{*}$ & 246 & 131 & 115 & $0.008^{*}$ \\
\hline & Present & 106 & 32 & 74 & & 49 & 16 & 33 & \\
\hline \multirow[t]{2}{*}{ Perineural invasion } & Absent & 321 & 168 & 153 & $<0.001^{*}$ & 275 & 141 & 134 & 0.066 \\
\hline & Present & 19 & 2 & 17 & & 20 & 6 & 14 & \\
\hline \multirow{2}{*}{$\begin{array}{l}\text { Mitotic rate (per } 10 \\
\text { high power fields) }\end{array}$} & $<10$ & 173 & 100 & 73 & $0.003^{*}$ & 139 & 79 & 60 & $0.023^{*}$ \\
\hline & $>=10$ & 167 & 70 & 97 & & 156 & 68 & 88 & \\
\hline
\end{tabular}

*Statisttically significant. 


\section{Correlations between immunoreactivity of RNF 128 and parameters in UTUC and UBUC}

RNF128 showed variable cytoplasmic expression in UC of both anatomic sites. As shown in Table 2, downregulated RNF128 expression in urothelial carcinoma of both sites was significantly correlated with stepwise increases in pT stage (Figure 2, p $<0.001$ for both UTUC and UBUC), nodal metastasis (UTUC, $\mathrm{p}<0.001$; UBUC, $\mathrm{p}=0.001$ ), high histological grade (UTUC, $\mathrm{p}<0.001$; UBUC, $\mathrm{p}=0.035$ ), vascular invasion (UTUC, $\mathrm{p}<0.001$; UBUC, $\mathrm{p}=0.008$ ), and high mitotic rate (UTUC, $\mathrm{p}=0.003 ; \quad \mathrm{UBUC}, \mathrm{p}=0.023$ ). Downregulation of RNF128 expression was significantly associated with perineural invasion in UTUC patients $(\mathrm{p}<0.001)$.

\section{Survival analysis of UTUC and UBUC}

The results of the univariate and multivariate analyses of association between clinical outcomes and several clinicopathological variables in both UTUC and UBUC patients are shown in Tables 3 and 4 , respectively. Univariate analysis indicated that poor DSS was significantly associated with tumor location

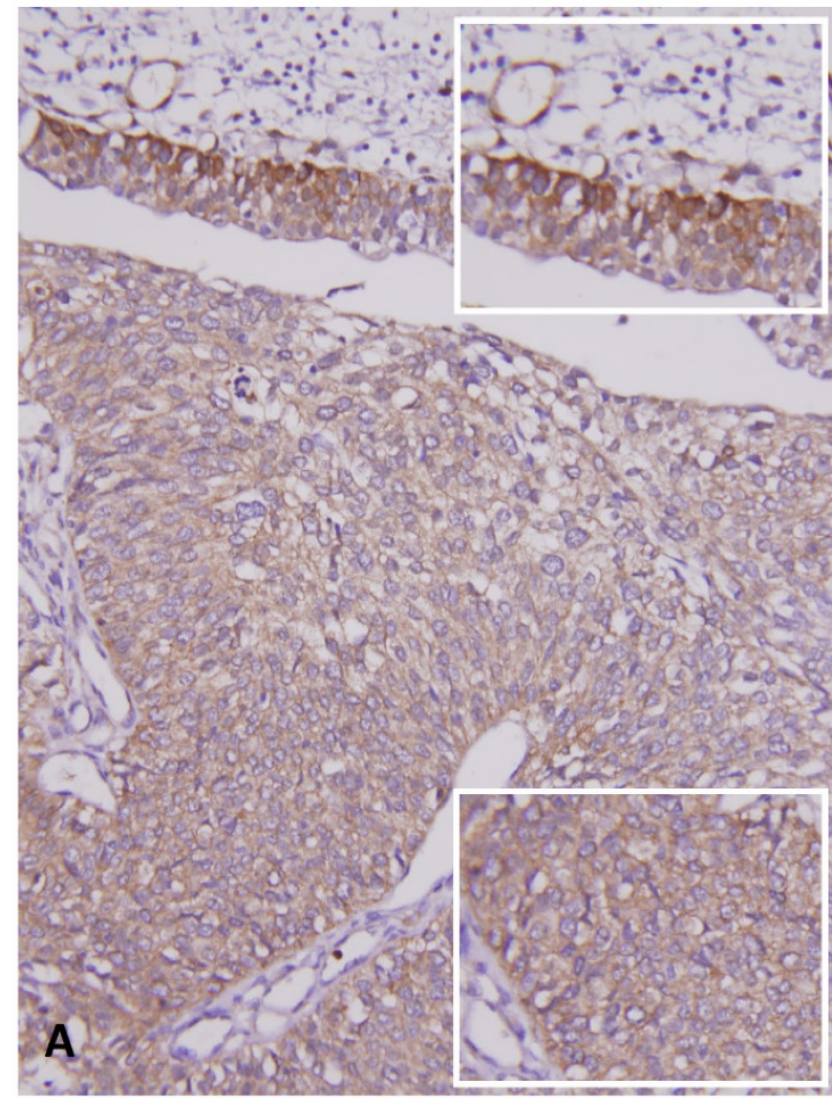

$(p=00079)$, multifocality $(p=0.0026)$, higher $p T$ status $(\mathrm{p}<0.0001)$, nodal metastasis $(\mathrm{p}<0.0001)$, high histological grade $(p=0.0215)$, vascular invasion $(p<0.0001)$ and perineural invasion $(p<0.0001)$ in UTUCs. Similar results were also found with MeFS, except for tumor location. In the UTUC group, only nodal metastasis (both $\mathrm{p}<0.001$ ) and perineural invasion (both $\mathrm{p}=0.002$ ) remained significantly correlated with worse DSS and MeFS in multivariate comparisons.

In the UBUC group, univariate analysis showed that poor DSS was significantly associated with higher $\mathrm{pT}$ status $(\mathrm{p}<0.0001)$, nodal metastasis $(p=0.0002)$, high histological grade $(p=0.0013)$, vascular invasion $(p=0.0024)$, perineural invasion $(p<0.0001)$ and high mitotic rate $(p<0.0001)$. The relevant findings for MeFS were similar. Multivariate analysis revealed that advanced pT stage was significantly associated with poor DDS and MeFS $(\mathrm{p}<0.0001$ and $\mathrm{p}=0.009$, respectively). High mitotic rate was also significantly correlated with poor DSS or MeFS ( $p=0.016$ and $p=0.028$, respectively) in the multivariate analysis.

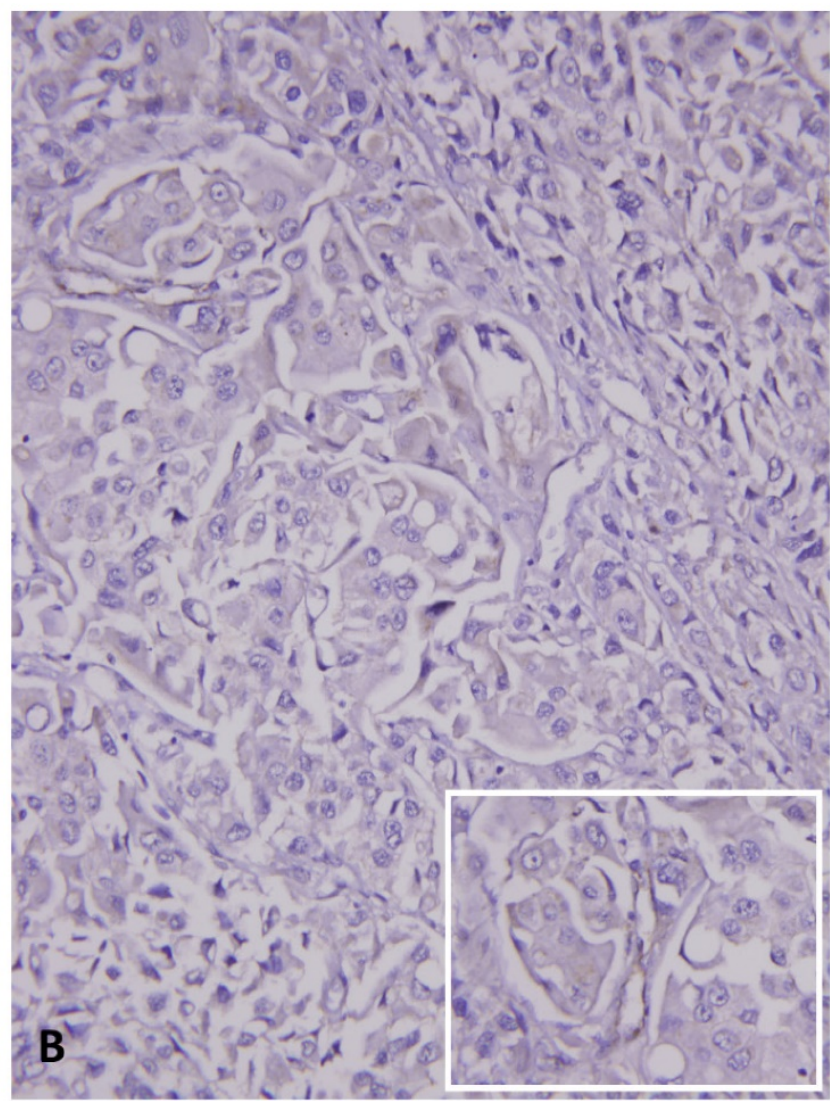

Figure 2. Immunohistochemical staining for RNF128 in representative case. (A) Intact expression of RNF128 in the non-neoplastic urothelium (upper and upper inset) is seen, compared with the weaker staining in the non-invasive urothelial carcinoma component (middle and lower, lower inset). (B) High-grade invasive urothelial carcinoma exhibits very low expression of RNF128 (inset). 
Table 3. Univariate log-rank and multivariate analyses for Disease-specific and Metastasis-free Survivals in upper urinary tract urothelial carcinoma.

\begin{tabular}{|c|c|c|c|c|c|c|c|c|c|c|c|c|}
\hline \multirow[t]{3}{*}{ Parameter } & \multirow[t]{3}{*}{ Category } & \multirow{3}{*}{$\begin{array}{l}\text { Case } \\
\text { No. }\end{array}$} & \multicolumn{5}{|c|}{ Disease-specific Survival } & \multicolumn{5}{|c|}{ Metastasis-free Survival } \\
\hline & & & \multicolumn{2}{|c|}{ Univariate analysis } & \multicolumn{3}{|c|}{ Multivariate analysis } & \multicolumn{2}{|c|}{ Univariate analysis } & \multicolumn{3}{|c|}{ Multivariate analysis } \\
\hline & & & No. of event & $\mathrm{p}$-value & R.R. & 95\% C.I. & p-value & No. of event & $\mathrm{p}$-value & R.R. & 95\% C.I. & p-value \\
\hline \multirow[t]{2}{*}{ Gender } & Male & 158 & 28 & 0.8286 & - & - & - & 32 & 0.7904 & - & - & - \\
\hline & Female & 182 & 33 & & - & - & - & 38 & & - & - & - \\
\hline \multirow[t]{2}{*}{ Age (years) } & $<65$ & 138 & 26 & 0.9943 & - & - & - & 30 & 0.8470 & - & - & - \\
\hline & $\geq 65$ & 202 & 35 & & - & - & - & 40 & & - & - & - \\
\hline \multirow[t]{3}{*}{ Tumor side } & Right & 177 & 34 & 0.7366 & - & - & - & 38 & 0.3074 & - & - & - \\
\hline & Left & 154 & 26 & & - & - & - & 32 & & - & - & - \\
\hline & Bilateral & 9 & 1 & & - & - & - & 0 & & - & - & - \\
\hline \multirow[t]{3}{*}{ Tumor location } & Renal pelvis & 141 & 24 & $0.0079^{*}$ & 1 & - & 0.560 & 31 & 0.0659 & - & - & - \\
\hline & Ureter & 150 & 22 & & 0.859 & $0.460-1.602$ & & 25 & & - & - & - \\
\hline & Renal pelvis \& ureter & 49 & 15 & & 1.845 & $0.491-6.935$ & & 14 & & - & - & - \\
\hline \multirow[t]{2}{*}{ Multifocality } & Single & 273 & 48 & $0.0026^{*}$ & 1 & - & 0.482 & 52 & $0.0127^{*}$ & 1 & - & $0.001^{*}$ \\
\hline & Multifocal & 62 & 18 & & 1.578 & $1.400-6.540$ & & 18 & & 2.790 & $1.563-4.980$ & \\
\hline \multirow[t]{3}{*}{$\begin{array}{l}\text { Primary tumor } \\
\text { (T) }\end{array}$} & $\mathrm{Ta}$ & 89 & 2 & $<0.0001^{*}$ & 1 & - & 0.130 & 4 & $\begin{array}{l}<0.0001 \\
*\end{array}$ & 1 & - & 0.135 \\
\hline & $\mathrm{T} 1$ & 92 & 9 & & 3.123 & $0.654-14.903$ & & 15 & & 3.216 & $0.678-15.265$ & \\
\hline & $\mathrm{T} 2-\mathrm{T} 4$ & 159 & 50 & & 4.633 & $1.005-21.347$ & & 51 & & 4.606 & $1.005-21.116$ & \\
\hline
\end{tabular}

*Statistically significant.

Table 4. Univariate log-rank and multivariate analyses for Disease-specific and Metastasis-free Survivals in urinary bladder urothelial carcinoma.

\begin{tabular}{|c|c|c|c|c|c|c|c|c|c|c|c|c|}
\hline \multirow[t]{3}{*}{ Parameter } & \multirow[t]{3}{*}{ Category } & \multirow{3}{*}{$\begin{array}{l}\text { Case } \\
\text { No. }\end{array}$} & \multicolumn{5}{|c|}{ Disease-specific Survival } & \multicolumn{5}{|c|}{ Metastasis-free Survival } \\
\hline & & & \multicolumn{2}{|c|}{ Univariate analysis } & \multicolumn{2}{|c|}{ Multivariate analysis } & \multirow[b]{2}{*}{ p-value } & \multicolumn{2}{|c|}{ Univariate analysis } & \multicolumn{3}{|c|}{ Multivariate analysis } \\
\hline & & & No. of event & $\mathrm{p}$-value & R.R. & 95\% C.I. & & No. of event & p-value & R.R. & 95\% C.I. & p-value \\
\hline \multirow[t]{2}{*}{ Gender } & Male & 216 & 41 & 0.4446 & - & - & - & 60 & 0.2720 & - & - & - \\
\hline & Female & 79 & 11 & & - & - & - & 16 & & - & - & - \\
\hline \multirow[t]{2}{*}{ Age (years) } & $<65$ & 121 & 17 & 0.1136 & - & - & - & 31 & 0.6875 & - & - & - \\
\hline & $\geq 65$ & 174 & 35 & & - & - & - & 45 & & - & - & - \\
\hline \multirow[t]{3}{*}{ Primary tumor $(\mathrm{T})$} & $\mathrm{Ta}$ & 84 & 1 & $<0.0001^{*}$ & 1 & - & $0.001^{*}$ & 4 & $<0.0001^{*}$ & 1 & - & $0.009^{*}$ \\
\hline & $\mathrm{T} 1$ & 88 & 9 & & 5.706 & $0.655-49.677$ & & 23 & & 4.754 & $1.430-15.800$ & \\
\hline & $\mathrm{T} 2-\mathrm{T} 4$ & 123 & 42 & & 19.576 & $2.351-163.031$ & & 49 & & 6.533 & $1.951-21.881$ & \\
\hline \multirow[t]{2}{*}{ Nodal metastasis } & Negative (N0) & 266 & 41 & $0.0002^{*}$ & 1 & - & 0.753 & 61 & $<0.0001^{*}$ & 1 & - & 0.075 \\
\hline & Positive (N1-N3) & 29 & 11 & & 1.121 & $0.524-2.169$ & & 15 & & 1.767 & $0.943-3.311$ & \\
\hline \multirow[t]{2}{*}{ Histological grade } & Low grade & 56 & 2 & $0.0013^{*}$ & 1 & - & 0.731 & 5 & $0.0007^{*}$ & 1 & - & 0.988 \\
\hline & High grade & 239 & 50 & & 1.312 & $0.279-6.170$ & & 71 & & 0.922 & $0.342-2.876$ & \\
\hline \multirow[t]{2}{*}{ Vascular invasion } & Absent & 246 & 37 & $0.0024^{*}$ & 1 & - & 0.254 & 54 & $0.0001^{*}$ & 1 & - & 0.790 \\
\hline & Present & 49 & 15 & & 0.666 & $0.332-1.339$ & & 22 & & 1.087 & $0.588-2.011$ & \\
\hline \multirow[t]{2}{*}{ Perineural invasion } & Absent & 275 & 44 & $0.0001^{*}$ & 1 & - & $0.037^{*}$ & 66 & $0.0007^{*}$ & 1 & - & 0.143 \\
\hline & Present & 20 & 8 & & 2.444 & $1.054-5.667$ & & 10 & & 1.740 & $0.829-3.654$ & \\
\hline \multirow{2}{*}{$\begin{array}{l}\text { Mitotic rate (per } 10 \\
\text { high power fields) }\end{array}$} & $<10$ & 139 & 12 & $<0.0001^{*}$ & 1 & - & $0.016^{*}$ & 23 & $<0.0001^{*}$ & 1 & - & $0.028^{*}$ \\
\hline & $>=10$ & 156 & 40 & & 2.292 & $1.169-4.491$ & & 53 & & 1.785 & $1.065-2.992$ & \\
\hline \multirow[t]{2}{*}{ RNF128 expression } & High & 147 & 12 & $<0.0001^{*}$ & 1 & - & $0.003^{*}$ & 26 & $0.0002^{*}$ & 1 & - & $0.036^{*}$ \\
\hline & Low & 148 & 20 & & 2.765 & $1.406-5.437$ & & 50 & & 1.712 & $1.035-2.830$ & \\
\hline
\end{tabular}

*Statistically significant.

\section{Prognostic value of RNF 128 expression in UCs}

Tables 3 and 4 show that low RNF128 cytoplasmic expression in univariate analysis was significantly related to poor DSS and MeFS in patients with either UTUC or UBUC (Figure 3). In addition, downregulated RNF128 expression still remained an independent prognosticator of poor DSS (UTUC, $\mathrm{p}=0.006$; UBUC, $\mathrm{p}=0.003$ ) and MeFS (UTUC, $\mathrm{p}=0.009$; UBUC, $\mathrm{p}=0.036$ ) in the multivariate comparisons.

\section{RNF 128 was downregulated in invasive urothelial cancer cells}

According to the Western blot and Real-time RT-PCR analysis, RNF128 transcript and protein were detected with high expression in RT4, which is a cell line derived from a non-invasive and low-grade urothelial cancer. While its expression was barely detected in invasive urothelial cancer cells including RT9, J82, and TSGH8301 (Figure 4). 

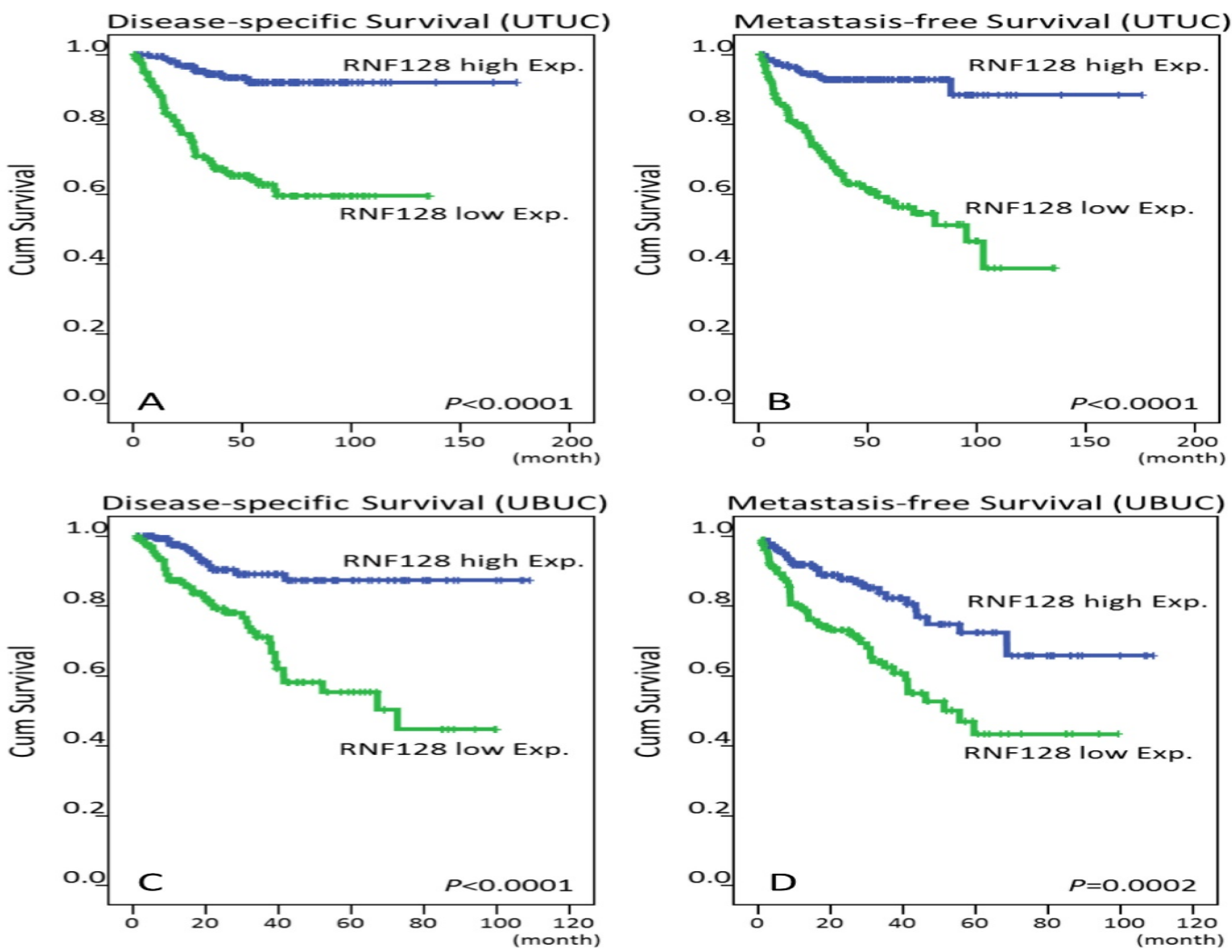

Figure 3. Survival analysis by Kaplan-Meier plots. Downregulation of RNF128 immunointensity confers poor disease-specific survival (DSS) and metastasis-free survival (MFS) in both upper tract urothelial carcinoma (UTUC, A and B) and urinary bladder urothelial carcinoma patients (UBUC, C and D).

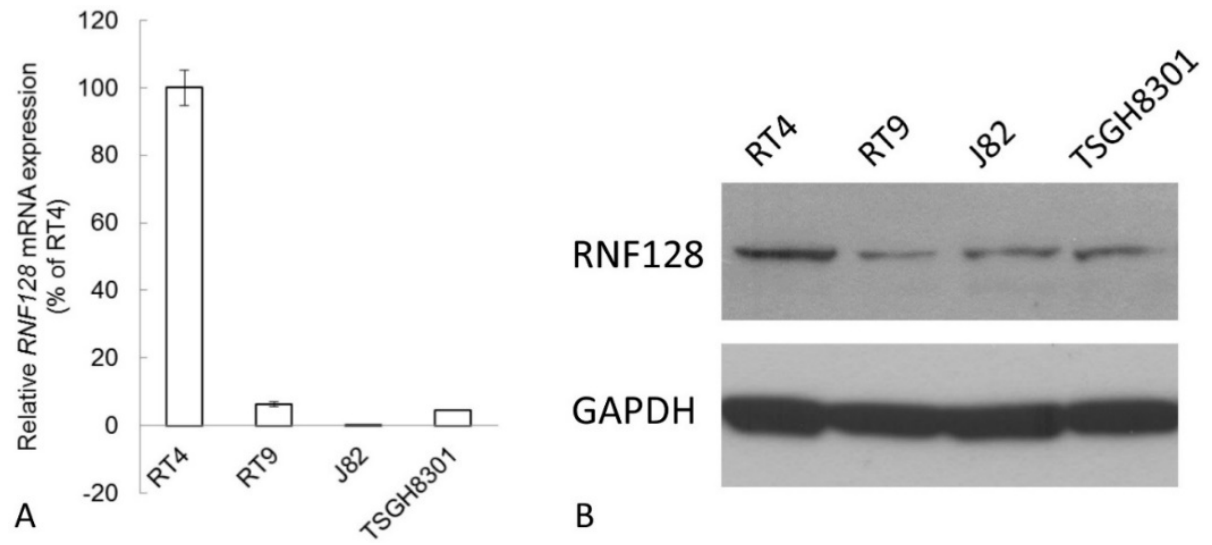

Figure 4. Endogenous RNF128 expression levels determined by quantitative RT-PCR (A) and western blotting assays (B). RNF128 expression is barely detected in invasive urothelial cancer cells including RT9, 82 , and TSGH8301 while its expression is high in RT4, a cell line derived from a non-invasive and low-grade urothelial cancer.

\section{Discussion}

Through data mining of the GEO data set GSE31684, we found that RNF128 expression was downregulated in UBUC compared to normal urothelium. Using immunohistochemistry, we showed that RNF128 expression in both UTUC and UBUC was significantly lower in advanced disease and was correlated with high tumor stage, high histological grade, high proliferation, and the presence of vascular and perineural invasion. Furthermore, downregulation of RNF128 was independently correlated with unfavorable outcomes in UC. To our knowledge, this is the first study to investigate the expression level and biological function of RNF128 in both UTUC and UBUC. Our findings indicated that downregulated RNF128 expression is a characteristic of poorly differentiated aggressive tumors. These results are consistent with those of Chen et al. [12]. Their unpublished data showed that RNF128 expression appeared to be downregulated in oral cancers through epigenetic modifications [12]. 
While the most reliable known prognostic factors for UBUC are tumor stage and histological grade, there have been multiple identified predictive markers for UTUC, including tumor multiplicity, anatomic ureteric location, tumor stage and high histological grade [4-11, 31-32]. However, these pathological features are not sufficient to accurately predict the evolution and progression of tumor invasion, especially for UTUC and UBUC. The utility and importance of biomarkers has been recognized and may help refine the understanding of the biological behavior of UTUC and UBUC, thereby improving the risk stratification and clinical management. Our study, for the first time, showed that low RNF128 expression was a promising molecular biomarker for the outcomes of both UTUC and UBUC. In addition, the results also indicated that UCs from both anatomical sites may share a common molecular pathway for tumorigenesis.

Alterations of the TP53 tumor suppressor gene play a central role in the carcinogenesis of many tumors, including UC [20-22]. The TP53 gene, located on the short arm of chromosome 17 (17p13.1), encodes a 393 amino acid protein (p53) that regulates the cell cycle, DNA repair, and apoptosis [21]. TP53 mutations result in a series of downstream effects, including decreased expression of p21 [12, 20-22]. TP53 mutations in UC are also related to higher tumor grade, advanced stage, and worse disease outcomes [21-22]. Downregulated expression of RNF128 was found to be involved in p53-induced apoptosis, playing an essential role in determining the cell fate, by Chen et al. [12]. The present study showed that RNF128 expression in UC was downregulated in samples with high tumor stages and histological grades and was associated with poorer patient outcomes. These results suggest that downregulation of RNF128 provides a growth advantage during UC tumorigenesis via p53 inactivation [33-35].

RNF128 functions as an E3 ubiquitin-protein ligase and has been characterized as a negative regulator of T-cell receptor responsiveness and cytokine production [12-13]. MDM2, a RING-type E3 of the ubiquitin proteasome system (UPS), is the main regulator of the p53 tumor suppressor protein, and targeting MDM2 by small molecules that prevent binding/ubiquitination of p53 has been of great research interest in recent years [36]. Four compounds that block the MDM2-p53 interactions are currently being assessed in phase I clinical trials [33-34]. RNF128, which has a similar role to MDM2 in the p53-MDM2 negative feedback loop, may be a promising therapeutic target through mediation of p53 ubiquitination [12, 36-37]. Therefore, it would be of interest to test this strategy of targeted treatment in
UC in the future.

Finally, there is an interesting issue with regard to the crosstalk between RNF128 and MDM2 for the regulation of p53 functions [12]. High activity of MDM2 has been observed in different cancers, leading to the reduction or loss of the p53 protein through ubiquitination, nuclear export, and transcriptional regulation [38-39]. TP53 mutation and MDM2 amplification were mutually exclusive events in the tumorigenesis of UC, both were correlated with greater recurrence and lower survival rate [40-41]. Although the p53-binding glycoprotein RNF128 was shown to function in a similar manner to that of MDM2 in the negative feedback loop, downregulation of RNF128 expression was observed in our present study as well as unpublished data by Chen et al. [12], in contrast to the usual overexpression of MDM2 in cancers. RNF128 appeared to be a key player in cell fate in p53-dependent apoptosis and cell cycle arrest [12]. Based on our present study results, we hypothesized that downregulated RNF128 expression exert a cooperative effect impacting tumor progression and survival in conjunction with p53-mediated pathway but not with MDM2 amplification. More research will be necessary to elucidate these effects.

Deregulated cell-cycle control and alterations in the ubiquitylation of cell-cycle regulators such as E3 ubiquitin ligases might lead to uncontrolled cell proliferation as well as genomic instability and have been shown to be implicated in many human malignancies [42-43]. Sahoo et al [13] found that the novel E3 ubiquitin ligase, RNF128, might play an important role in controlling Th2 development by targeting signal transducer and activator of transcription 6 (STAT6) [13]. GATA binding protein 3 (GATA3), a transcriptional factor downstream of STAT6, was also suggested to regulate RNF128 expression among those participating in Th2 cells depending interleukin (IL)-4 components [13, 44]. Previous studies have identified STAT6 as a crucial cell differentiation modulator in human breast cancer cells through IL-4-mediated growth inhibition and induction of apoptosis [45-46]. Followed by the utility in the differentiation of mammary epithelia, GATA3 has been identified as novel marker for the diagnosis of urothelial carcinoma [47]. Moreover, GATA3 was found to be downregulated in higher grade breast cancer and invasive bladder cancer [47-48]. In the present study, we showed that downregulation of RNF128 expression was correlated with higher tumor stage in invasive urothelial carcinoma. This tendency was in parallel with GATA3 expression seen in previous studies. We therefore supposed that downregulated GATA3 expression resulted in lower 
RNF128 expression in higher grade invasive urothelial carcinoma, which may be involved by STAT6 or not. Further investigation would be addressed on a need bases. Additionally, endogenous RNF128 expression was shown to participate in the cell cycle and promotes the proliferation of primary naive CD4 T-cells as aforementioned findings [13-14]. Thus, it would be of interest to study the role of RNF128 during T-cell unresponsiveness and whether it may serve as a possible target in immunotherapy for cancer treatment [13-14].

Moreover, downregulation of RNF128 expression is an independent predictor for UTUC and UBUC. Our findings suggest that RNF128 is not only a diagnostic biomarker but also a prognostic biomarker in UC. Further evaluation to elucidate the biological significance of dysregulated RNF128 expression in UC is needed to explore the potential of RNF128-targeted therapy in UC.

\section{Acknowledgements}

This study is supported by Chi Mei Medical Center, Liouying Campus (CLFHR10534). This study was also supported by grants from Kaohsiung Medical University "Aim for the Top Universities" (KMU-TP104E31, KMU-TP105G00, KMU-TP105G01, KMU-TP105G02), the health and welfare surcharge of tobacco products, Ministry of Health and Welfare (MOHW105-TDU-B-212-134007), Ministry of Science and Technology (MOST103-2314-B-037-059-MY3, MOST 104-2314-B-037-050-MY3), and Kaohsiung Medical University Hospital (KMUH101-1R42, KMUH103-3M37).

\section{Competing Interests}

The authors have declared that no competing interest exists.

\section{References}

1. Holger Moch, Peter A. Humphrey, Thomas M. Ulbright, Victor E. Reuter (Eds): WHO Classification of Tumours of the Urinary System and Male Genital Organs (4th edition). IARC: 2016.

2. Antoni S, Ferlay J, Soerjomataram I, Znaor A, Jemal A, Bray F. Bladder Cancer Incidence and Mortality: A Global Overview and Recent Trends. Eur Urol. 2016 Jun 28. pii: S0302-2838(16)30280-9. doi: 10.1016/j.eururo.2016.06.010. [Epub ahead of print]

3. Siegel R, Naishadham D, Jemal A. Cancer statistics, 2012. CA Cancer J Clin. 2012; 62:10-29.

4. Green DA, Rink M, Xylinas E, Matin SF, Stenzl A, Roupret M, et al. Urothelial carcinoma of the bladder and the upper tract: disparate twins. J Urol. 2013;189:1214-21.

5. Yates DR, Catto JW. Distinct patterns and behaviour of urothelial carcinoma with respect to anatomical location: how molecular biomarkers can augment clinico-pathological predictors in upper urinary tract tumours. World J Urol. 2013;31:21-9.

6. Kim M, Jeong CW, Kwak $\mathrm{C}, \mathrm{Kim} \mathrm{HH}, \mathrm{Ku} \mathrm{JH}$. Are urothelial carcinomas of the upper urinary tract a distinct entity from urothelial carcinomas of the urinary bladder? Behavior of urothelial carcinoma after radical surgery with respect to anatomical location: a case control study. BMC Cancer. 2015;15:149.

7. Zhang Z, Furge KA, Yang XJ, Teh BT, Hansel DE. Comparative gene expression profiling analysisof urothelial carcinoma of the renal pelvis and bladder. BMC Med Genomics. 2010;3:58.
8. Chang IW, Lin VC, Wu WJ, Liang PI, Li WM, Yeh BW, et al. Complement Component 1, s Subcomponent Overexpression is an Independent Poor Prognostic Indicator in Patients with Urothelial Carcinomas of the Upper Urinary Tract and Urinary Bladder. J Cancer. 2016;7:1396-405.

9. Chang IW, Li CF, Lin VC, He HL, Liang PI, Wu WJ, et al. Prognostic Impact of Thrombospodin-2 (THBS2) Overexpression on Patients with Urothelial Carcinomas of Upper Urinary Tracts and Bladders. J Cancer. 2016;7:1541-9.

10. Azémar MD, Comperat E, Richard F, Cussenot O, Rouprêt M. Bladder recurrence after surgery for upper urinary tract urothelial cell carcinoma: frequency, risk factors, and surveillance. Urol Oncol. 2011;29:130-6.

11. Sanderson KM, Rouprêt M. Upper urinary tract tumour after radical cystectomy for transitional cell carcinoma of the bladder: an update on the risk factors, surveillance regimens and treatments. BJU Int. 2007;100:11-6.

12. Chen YC, Chan JY, Chiu YL, Liu ST, Lozano G, Wang SL, et al. Grail as a molecular determinant for the functions of the tumor suppressor p53 in tumorigenesis. Cell Death Differ. 2013;20:732-43.

13. Sahoo A, Alekseev A, Obertas L, Nurieva R. Grail controls Th2 cell development by targeting STAT6 for degradation. Nat Commun. 2014;5:4732.

14. Whiting CC, Su LL, Lin JT, Fathman CG. GRAIL: a unique mediator of CD4 T-lymphocyte unresponsiveness. FEBS J. 2011;278:47-58.

15. May P, May E. Twenty years of p53 research: structural and functional aspects of the p53 protein. Oncogene. 1999;18:7621-36.

16. Meek DW. Tumour suppression by p53: a role for the DNA damage response? Nat Rev Cancer. 2009;9:714-23.

17. Zuckerman V, Wolyniec K, Sionov RV, Haupt S, Haupt Y. Tumour suppression by p53: the importance of apoptosis and cellular senescence. J Pathol. 2009;219:3-15.

18. Hanahan D, Weinberg RA. Hallmarks of cancer: the next generation. Cell. 2011;144:646-74

19. Zhu J, Sammons MA, Donahue G, Dou Z, Vedadi M, Getlik M, et al. Gain-of-function p53 mutants co-opt chromatin pathways to drive cancer growth. Nature. 2015;525:206-11.

20. Cote RJ, Dunn MD, Chatterjee SJ, Stein JP, Shi SR, Tran QC, et al. Elevated and absent $\mathrm{pRb}$ expression is associated with bladder cancer progression and has cooperative effects with p53. Cancer Res. 1998;58:1090-4.

21. Cheng L, Zhang S, MacLennan GT, Williamson SR, Lopez-Beltran A, Montironi R. Bladder cancer: translating molecular genetic insights into clinical practice. Hum Pathol. 2011;42:455-81.

22. Senol S, Yildirim A, Ceyran B, Uruc F, Zemheri E, Ozkanli S, et al. Prognostic significance of survivin, $\beta$-catenin and p53 expression in urothelial carcinoma. Bosn J Basic Med Sci. 2015;15:7-14.

23. Liang PI, Wang $\mathrm{YH}, \mathrm{Wu} \mathrm{TF}, \mathrm{Wu} W \mathrm{~W}$, Liao AC, Shen $\mathrm{KH}$, et al. IGFBP-5 overexpression as a poor prognostic factor in patients with urothelial carcinomas of upper urinary tracts and urinary bladder. J Clin Pathol. 2013;66:573-82.

24. Fan EW, Li CC, Wu WJ, Huang CN, Li WM, Ke HL, et al. FGF7 Over Expression is an independent prognosticator in patients with urothelial carcinoma of the upper urinary tract and bladder. J Urol. 2015;194:223-9.

25. Chang IW, Wang YH, Wu WJ, Liang PI, Li WM, Yeh BW, et al. Necdin Overexpression Predicts Poor Prognosis in Patients with Urothelial Carcinomas of the Upper Urinary Tract and Urinary Bladder. J Cancer. 2016;7:304-13

26. Lee $Y Y, W u$ WJ, Huang CN, Li CC, Li WM, Yeh BW, et al. CSF2 Overexpression Is Associated with STAT5 Phosphorylation and Poor Prognosis in Patients with Urothelial Carcinoma. J Cancer. 2016;7:711-21.

27. Ma LJ, Wu WJ, Wang YH, Wu TF, Liang PI, Chang IW, et al. SPOCK1 Overexpression Confers a Poor Prognosis in Urothelial Carcinoma. J Cancer. 2016:7:467-76.

28. Chang IW, Wu WJ, Wang YH, Wu TF, Liang PI, He HL, et al. BCAT1 overexpression is an indicator of poor prognosis in patients with urothelial carcinomas of the upper urinary tract and urinary bladder. Histopathology. 2016;68:520-32.

29. Li CF, Wu WJ, Wu WR, Liao YJ, Chen LR, Huang CN, et al. The cAMP responsive element binding protein 1 transactivates epithelial membrane protein 2, a potential tumor suppressor in the urinary bladder urothelial carcinoma. Oncotarget. 2015;6:9220-39.

30. Wang YH, Wu WJ, Wang WJ, Huang HY, Li WM, Yeh BW, et al. CEBPD amplification and overexpression in urothelial carcinoma: a driver of tumor metastasis indicating adverse prognosis. Oncotarget. 2015;6:31069-84.

31. Catto JW, Yates DR, Rehman I, Azzouzi AR, Patterson J, Sibony M, et al. Behavior of urothelial carcinoma with respect to anatomical location. J Urol. 2007;177:1715-20.

32. Kim HS, Piao S, Moon KC, Jeong CW, Kwak C, Kim HH, et al. Adjuvant Chemotherapy Correlates with Improved Survival after Radical Cystectomy in Patients with pT3b (Macroscopic Perivesical Tissue Invasion) Bladder Cancer. J Cancer. 2015;6:750-8.

33. Fan YH, Cheng J, Vasudevan SA, Dou J, Zhang H, Patel RH, et al. USP7 inhibitor P22077 inhibits neuroblastoma growth via inducing p53-mediated apoptosis. Cell Death Dis. 2013;4:e867.

34. Mazza D, Infante P, Colicchia V, Greco A, Alfonsi R, Siler M, et al. PCAF ubiquitin ligase activity inhibits Hedgehog/Gli1 signaling in p53-dependent response to genotoxic stress. Cell Death Differ. 2013;20:1688-97.

35. Sermeus A and Michiels C. Reciprocal influence of the p53 and the hypoxic pathways. Cell Death Dis. 2011;2:e164 
36. Landré V, Rotblat B, Melino S, Bernassola F, Melino G. Screening for E3-ubiquitin ligase inhibitors: challenges and opportunities. Oncotarget. 2014;5:7988-8013.

37. Hoe KK, Verma CS, Lane DP. Drugging the p53 pathway: understanding the route to clinical efficacy. Nature reviews Drug discovery. 2014;13:217-36.

38. Oliner JD, Kinzler KW, Meltzer PS, George DL, Vogelstein B. Amplification of a gene encoding a p53-associated protein in human sarcomas. Nature. 1992;358:80-3

39. Momand J, Wu HH, Dasgupta G. MDM2-master regulator of the p53 tumor suppressor protein. Gene. 2000;242:15-29.

40. Lu ML, Wikman F, Orntoft TF, Charytonowicz E, Rabbani F, Zhang Z, et al. Impact of alterations affecting the p53 pathway in bladder cancer on clinical outcome, assessed by conventional and array-based methods. Clin Cancer Res. 2002;8:171-9.

41. Bagrodia A, Cha EK, Sfakianos JP, Zabor EC, Bochner BH, Al-Ahmadie HA, et al. Genomic Biomarkers for the Prediction of Stage and Prognosis of Upper Tract Urothelial Carcinoma. J Urol. 2016;195:1684-9.

42. Nakayama KI, Nakayama K. Ubiquitin ligases: cell-cycle control and cancer. Nat Rev Cancer. 2006;6:369-81.

43. Snoek BC, de Wilt LH, Jansen G, Peters GJ. Role of E3 ubiquitin ligases in lung cancer. World J Clin Oncol. 2013;4:58-69

44. Lee GR, Fields PE, Flavell RA. Regulation of IL-4 gene expression by distal regulatory elements and GATA-3 at the chromatin level. Immunity. 2001;14:447-59.

45. Gooch JL, Christy B, Yee D. STAT6 mediates interleukin-4 growth inhibition in human breast cancer cells. Neoplasia. 2002;4:324-31.

46. Wei M, Liu B, Gu Q, Su L, Yu Y, Zhu Z. Stat6 cooperates with Sp1 in controlling breast cancer cell proliferation by modulating the expression of p21(Cip1/WAF1) and p27 (Kip1). Cell Oncol (Dordr). 2013;36:79-93.

47. Miyamoto H, Izumi K, Yao JL, Li Y, Yang Q, McMahon LA, et al. GATA binding protein 3 is down-regulated in bladder cancer yet strong expression is an independent predictor of poor prognosis in invasive tumor. Hum Pathol. 2012;43:2033-40.

48. Dydensborg AB, Rose AA, Wilson BJ, Grote D, Paquet $\mathrm{M}$, Giguère $\mathrm{V}$, et al. GATA3 inhibits breast cancer growth and pulmonary breast cancer metastasis. Oncogene. 2009;28:2634-42. 